\title{
Diagnosi per immagini istologiche, immunoistochimiche e sonografiche di un raro caso di plasmocitoma scarsamente differenziato della tiroide
}

\author{
Loredana Lorusso $^{1} \cdot$ Laura Agate $^{1} \cdot$ Clara Ugolini $^{2} \cdot$ Rossella Elisei $^{1}$
}

Accettato: 26 aprile 2020 / Pubblicato online: 26 ottobre 2020

(c) The Author(s) 2020

Un uomo di 90 anni giungeva alla nostra osservazione lamentando disfagia e dispnea da alcuni giorni, con una tumefazione di circa $10 \mathrm{~cm}$ a livello cervicale anteriore, dura alla palpazione, adesa ai piani sottostanti e ricoperta da cute normale.

L'ecografia del collo evidenziava, in una ghiandola tiroidea del volume di 88 millilitri circa, un nodulo solido che sostituiva completamente il lobo destro (Fig. 1) e tre noduli ipoecogeni a carico dell'istmo e del lobo sinistro. In sede laterocervicale erano presenti numerose linfoadenopatie, la maggiore a destra di $22 \times 36 \times 47 \mathrm{~mm}$.

Il paziente veniva sottoposto a biopsia con ago tranciante del lobo tiroideo destro.

L'esame istologico era conclusivo per "parenchima tiroideo con diffusa infiltrazione di neoplasia scarsamente differenziata. Immunofenotipo: cromogranina, sinaptofisina, CD56, tireoglobulina, TTF1, PAX8, CKCAM5.2, CK7, CDX2, CD79a negativo; CD138: positivo". I reperti morfologici e immunoistochimici risultavano orientativi per plasmocitoma (Fig. 2).

Due giorni dopo la nostra valutazione, un rapido peggioramento della dispnea e della disfagia richiedevano l'ospedalizzazione del paziente che, dopo un giorno di degenza, decedeva.

Il plasmocitoma è una neoplasia caratterizzata dalla proliferazione di un clone di cellula B in grado di produrre immunoglobuline. Le presentazioni cliniche di tale neoplasia vanno da una condizione asintomatica, senza coinvolgi-

L. Lorusso

lorussoloredana@hotmail.it

1 Dipartimento di Medicina Clinica e Sperimentale, Sezione di Endocrinologia, Università di Pisa, Pisa, Italia

2 Dipartimento di medicina di Laboratorio, U.O. Anatomia Patologica $3^{\circ}$ Universitaria, Azienda Ospedaliero-Universitaria Pisana, Pisa, Italia

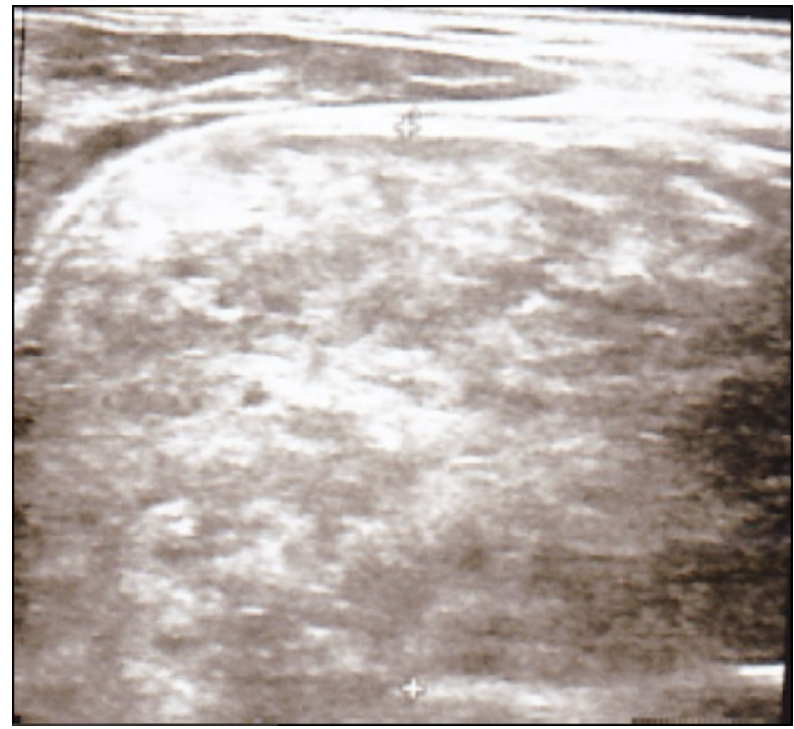

Fig. 1 Aspetto ecografico del nodulo solido che sostituiva completamente il lobo tiroideo destro. Il nodulo appariva lievemente ipoecogeno e marcatamente disomogeneo e tendente ad affondare nel giugulo

mento di organi, a una condizione caratterizzata da singole/multiple lesioni in siti midollari o extramidollari. Il 3\% dei plasmocitomi insorge in siti extramidollari e, sebbene questi prediligano la mucosa del tratto respiratorio superiore, è stata descritta una localizzazione primaria in varie sedi e tra queste la tiroide $[1,2]$.

La Figura 1 mostra l'aspetto ecografico del lobo tiroideo destro del caso presentato: un voluminoso nodulo lievemente ipoecogeno e marcatamente disomogeneo sostituiva completamente il lobo, affondando nel giugulo. La Figura 2 mostra l'immunoistochimica dell'istologia del caso presentato (ingrandimento $2 \times$ ): le plasmacellule tumorali risultavano positive per l'anti-plasmacellule (VS38C clone) (Fig. 2a) e CD138 (Fig. 2b). 
Fig. 2 a, b Immunoistochimica dell'istologia del caso presentato: le plasmacellule tumorali risultavano positive per l'anti-plasmacellule (VS38C clone) (a) e CD138 (b) (ingrandimento $2 \times$ )

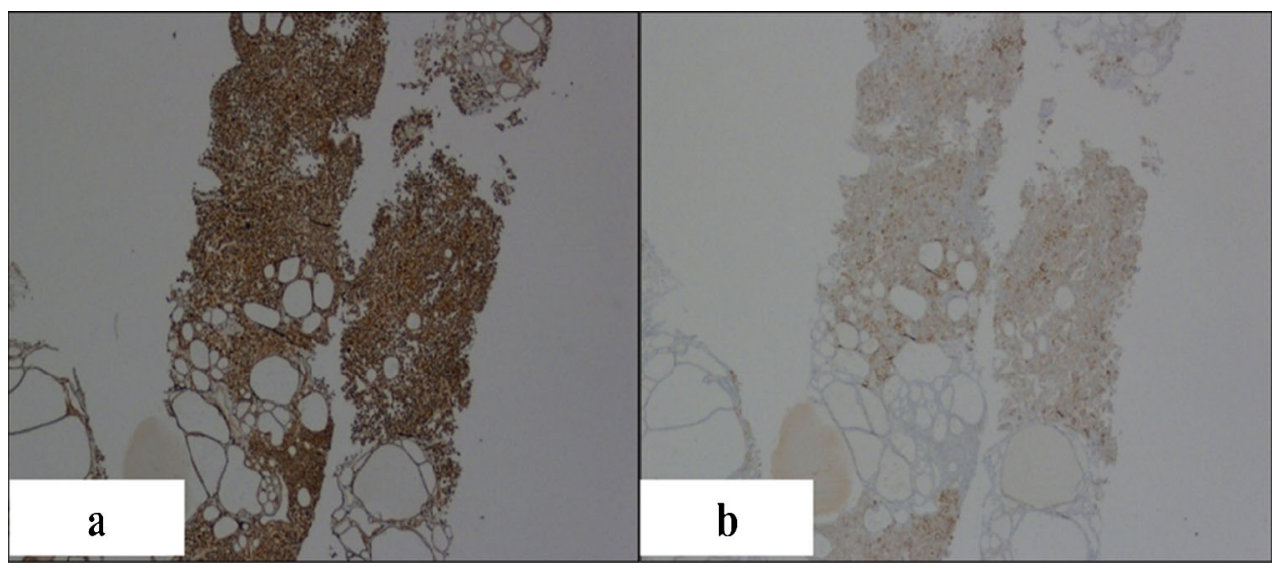

La diagnosi di questo raro caso di localizzazione tiroidea di un plasmocitoma indifferenziato ad esito rapidamente fatale si è basata sul rilievo ecografico (Fig. 1) di un nodulo solido che si sostituiva completamente al lobo tiroideo destro e di alcune linfoadenopatie laterocervicali, ipoecogene con numerosi setti iperecogeni interni. L'indagine istologica mostrava un parenchima tiroideo micro-macrofollicolare densamente infiltrato da una popolazione neoplastica costituita da elementi plasma cellulari con immunofenotipo positivo per VS38C clone (Fig. 2a) e CD138 (Fig. 2b). I dati immunoistochimici hanno, pertanto, consentito di formulare la rara diagnosi.

Funding Note Open access funding provided by Università di Pisa within the CRUI-CARE Agreement.

Conflitto di interesse Gli autori Loredana Lorusso, Laura Agate, Clara Ugolini e Rossella Elisei dichiarano di non avere conflitti di interesse.

Consenso informato Il consenso informato del paziente era stato preliminarmente ottenuto.

Studi sugli animali Gli autori di questo articolo non hanno eseguito studi sugli animali.
Nota della casa editrice Springer Nature rimane neutrale in riguardo alle rivendicazioni giurisdizionali nelle mappe pubblicate e nelle affiliazioni istituzionali.

Open Access This article is licensed under a Creative Commons Attribution 4.0 International License, which permits use, sharing, adaptation, distribution and reproduction in any medium or format, as long as you give appropriate credit to the original author(s) and the source, provide a link to the Creative Commons licence, and indicate if changes were made. The images or other third party material in this article are included in the article's Creative Commons licence, unless indicated otherwise in a credit line to the material. If material is not included in the article's Creative Commons licence and your intended use is not permitted by statutory regulation or exceeds the permitted use, you will need to obtain permission directly from the copyright holder. To view a copy of this licence, visit http://creativecommons.org/licenses/by/4.0/.

\section{Bibliografia}

1. Sahu K, Singh P, Malhotra P, Srinivasan R (2019) Thyroid plasmacytoma: a rare cause of hoarseness of voice. Indian J Nucl Med 34:78-80

2. Gochhait D, Govindarajalou R, Kar R et al (2019) Plasmacytoma of thyroid clinically and morphologically mimicking primary thyroid carcinoma. Cytopathology 30:113-116 